\title{
ON SOME FUNDAMENTAL PROBLEMS IN CLUSTER SET THEORY
}

\author{
J. A. EIDSWICK
}

\begin{abstract}
An attempt to characterize the local behavior of arbitrary functions of two real variables in terms of their cluster sets along various approach curves leads to two main problems: (1) Finding a "small" family $\Gamma_{0}$ of approach curves such that cluster sets along $\Gamma_{0}$ determine total cluster sets; (2) Finding a "large" family of approach curves along which cluster sets can be preassigned. A nice solution of (1) is found and (2) is partially solved. Conjectures are made concerning a link between (2) and sets which are always of the first category.
\end{abstract}

1. Introduction. If $f$ is a real-valued function defined on the real line $R$, the sets $C^{-}(f)$ and $C^{+}(f)$ of left- and right-hand limit points of $f$ at 0 (say) are closed. Conversely, if $C^{-}$and $C^{+}$are closed sets, Bettazzi [1, p. 173] constructs a function $f$ such that $C^{-}(f)=C^{-}$and $C^{+}(f)=C^{+}$(for a proof in English, see [4, p. 319]). Thus the local behavior of $f$ is more or less characterized by pairs of closed sets. An attempt to extend this characterization to the two-dimensional case leads to these problems:

I. Find a "small" subset $\Gamma_{0}$ of the set $\Gamma$ of all approach curves such that for each function $f,\left\{C\left(f, \gamma_{0}\right): \gamma_{0} \in \Gamma_{0}\right\}$ determines $C(f, \gamma)$ for all $\gamma$ on $\Gamma$.

II. Find a "large" subset $\Gamma_{0}$ of $\Gamma$ such that to any collection $\left\{C\left(\gamma_{0}\right)\right.$ : $\left.\gamma_{0} \in \Gamma_{0}\right\}$ of closed sets there corresponds a function $f$ such that $C\left(f, \gamma_{0}\right)=$ $C\left(\gamma_{0}\right)$ for all $\gamma_{0}$ in $\Gamma_{0}$.

Here $C(f, \gamma)$ denotes the cluster set of $f$ at 0 along the approach curve $\gamma$, i.e., $C(f, \gamma)=\left\{\lambda\right.$ : there exists a sequence $\left\{P_{n}\right\}$ of distinct points on $\gamma$ such that $P_{n} \rightarrow 0$ and $\left.f\left(P_{n}\right) \rightarrow \lambda\right\}$. (For the general theory of cluster sets, see [2] and [6].) By an approach curve will be meant the graph or any rotation of the graph of a real-valued continuous function on $[0, \infty)$. Adjectives "convex", "continuously differentiable", etc., when applied to approach curves are defined in terms of the reference function. Thus, a convex approach curve is the graph or rotation of the graph of a convex continuous function on $[0, \infty)$. (A straight line is regarded as a special case of

Received by the editors July $24,1972$.

AMS (MOS) subject classifications (1970). Primary 26A15; Secondary 28A05.

Key words and phrases. Cluster sets, approach curves, truncations, sets which are always of the first category.

(c) American Mathematical Society 1973 
a convex approach curve.) Two approach curves will be identified if they coincide in some neighborhood of the origin.

Ideally, Problems I and II would have a common solution. However, we will see that this is asking for too much.

2. Solution of Problem I. In a strict sense, Problem I has no solution. However, by properly interpreting the word "determines" we are able to get a most satisfying solution.

THEOREM 1. If $\Gamma_{0}$ is a proper subset of $\Gamma$ and $\gamma$ is an element of $\Gamma-\Gamma_{0}$, then there exist functions $f$ and $g$ such that $C\left(f, \gamma_{0}\right)=C\left(g, \gamma_{0}\right)$ for every $\gamma_{0}$ in $\Gamma_{0}$ but $C(f, \gamma) \neq C(g, \gamma)$.

Proof. Let $(A, B)$ be a decomposition of the plane $\left(A \cup B=R^{2}\right.$, $A \cap B=\varnothing$ ) such that $A$ and $B$ contain no arcs. (Proof that such a decomposition exists: Well order the set of all arcs in such a way that any initial segment of the ordering has cardinality less than the cardinality of the continuum; then, observing that the set of all arcs of the plane is equivalent to the set of all points of the plane, construct disjoint sets $A_{0}$ and $B_{0}$ inductively so that both $A_{0}$ and $B_{0}$ intersect every arc; finally, set $A=A_{0}$ and $B=R^{2}-A$.) For $P \notin \gamma$ define

$$
\begin{aligned}
f(P)=g(P)=0 & & \text { if } P \in A, \\
=1 & & \text { if } P \in B
\end{aligned}
$$

and for $P \in \gamma$ define $f(P)=0$ and $g(P)=1$. Then $C\left(f, \gamma_{0}\right)=\{0,1\}=C\left(g, \gamma_{0}\right)$ for all $\gamma_{0}$ in $\Gamma_{0}$ but $C(f, \gamma)=\{0\}$ and $C(g, \gamma)=\{1\}$.

Henceforth, we will interpret "determines" in Problem I to mean only that

for every $\gamma$ in $\Gamma$.

$$
C(f, \gamma) \subset \bigcup_{\gamma_{0} \in \Gamma_{0}} C\left(f, \gamma_{0}\right)
$$

THEOREM 2. If $\Gamma_{0}$ is the set of all convex differentiable approach curves, then (*) holds for any function $f$ and any $\gamma \in \Gamma$. However, if $\Gamma_{0}$ is only the set of all (at least) twice differentiable approach curves, then (*) fails for some function $f$ and some $\gamma$ in $\Gamma$.

The result is sharp because any convex differentiable approach curve is continuously differentiable (see, e.g., [3]).

LEMMA. If $\left\{P_{n}\right\}$ is any sequence of distinct points converging to 0 , then there exists a convex differentiable approach curve $\gamma_{0}$ and a subsequence $\left\{\boldsymbol{P}_{n_{k}}\right\}$ of $\left\{\boldsymbol{P}_{n}\right\}$ such that $\boldsymbol{P}_{n_{k}} \in \gamma_{0}$ for all $k$. On the other hand, the approach curve $\gamma$ defined by $y=x^{3 / 2}, x \geqq 0$, has the property that there is no twice 
differentiable approach curve which intersects $\gamma$ on a sequence $\left\{P_{n}\right\}$ of distinct points converging to 0.

The lemma is a modification of a result due to A. Rosenthal [7].

Proof of Lemma. The first statement is equivalent to the first part of Theorem 2 of [7]. For the second statement, let $\gamma_{0}$ be a differentiable approach curve which intersects $\gamma$ on an infinite sequence of points converging to 0 . We show that $\gamma_{0}$ cannot be twice differentiable by showing that it cannot have a second derivative at 0 .

Even though $\gamma_{0}$ contains a sequence of points converging to 0 in the first quadrant, it may happen that it is impossible to represent it by a singlevalued function. However, if $\gamma_{0}$ is rotated through a suitable acute angle $\theta$, it can be represented in some positive half-neighborhood of 0 by a differentiable function $\phi_{0}(x)$. Moreover, when $\gamma$ is rotated through the same angle, it can be represented in some positive half-neighborhood of 0 by a function $\phi(x)$ having these properties:

(i) $\phi(0)=0, \phi^{\prime}(0)=\tan \theta$,

(ii) $\left(\phi^{\prime}(x)-\tan \theta\right) / x \rightarrow+\infty$ as $x \downarrow 0$,

(iii) $\phi\left(x_{n}\right)=\phi_{0}\left(x_{n}\right)$ for some sequence $x_{n} \downarrow 0$.

By (i) and (iii), $\phi_{0}^{\prime}(0)=\tan \theta$ and it follows from (ii) that $\left[\phi_{0}^{\prime}(x)-\tan \theta\right] / x$ cannot be bounded. (If it were, we would have $\phi^{\prime}(x)>\phi_{0}^{\prime}(x)$ and hence $\phi(x)>\phi_{0}(x)$ in some positive half-neighborhood of 0 contrary to (iii).) Hence, $\phi_{0}^{\prime \prime}(0)$ cannot exist. (Cf. the proof of Theorem 2 of [7].)

Proof of Theorem 2. If $\lambda \in C(f, \gamma)$, then there exists a sequence $\left\{\boldsymbol{P}_{n}\right\}$ of distinct points on $\gamma$ such that $P_{n} \rightarrow 0$ and $f\left(P_{n}\right) \rightarrow \lambda$. It then follows from the lemma that $\lambda \in C\left(f, \gamma_{0}\right)$ for some convex differentiable approach curve $\gamma_{0}$.

For the second part, let $\gamma$ be as in the lemma and define $f(P)=1$ for $P \in \gamma, f(P)=0$ for $P \notin \gamma$. Then $C(f, \gamma)=\{1\}$ and, by the lemma, $C\left(f, \gamma_{0}\right)=$ $\{0\}$ for every twice differentiable approach curve $\gamma_{0}$.

3. Partial solution of Problem II. We start by looking at some simple examples. First, Bettazzi's construction shows that any one point family $\Gamma_{0}=\left\{\gamma_{0}\right\}$ is a solution. Indeed, the same reasoning shows that any nonintersecting family such as the family of all radial approaches is a solution. It is also clear that any family that intersects itself at only a finite number of points is a solution. However, a family that intersects itself infinitely many times need not be a solution. This is a consequence of Theorem 3 (below).

Two approach curves will be called comparable if they are disjoint in some deleted neighborhood of the origin. A family $\Gamma_{0}$ of approach curves will be called comparable if any two curves in $\Gamma_{0}$ are comparable. 
THeORem 3. Any family which is a solution of Problem II must be comparable.

It follows that there exist families of convex differentiable approach curves which are not solutions of Problem II. In fact, if $\psi(x)=$ $(\sin \pi / x) e^{-1 / x}$ for $x>0, \psi(0)=0$, and $M$ is a bound for $\psi^{\prime \prime}$ on $[0, \infty)$, then the approach curves $\gamma_{1}$ and $\gamma_{2}$ defined for $x \geqq 0$ by $y_{1}(x)=M x^{2}$ and $y_{2}(x)=$ $M x^{2}+\psi(x)$, respectively, are noncomparable, infinitely-differentiable, and convex.

Proof of Theorem 3. If $\gamma_{1}$ and $\gamma_{2}$ are noncomparable approach curves, they intersect on a sequence $\left\{P_{n}\right\}$ of distinct points converging to 0 . Hence, $C\left(f, \gamma_{1}\right) \cap C\left(f, \gamma_{2}\right) \neq \varnothing$ for every function $f$.

THEOREM 4. A comparable family $\Gamma_{0}$ of approach curves is a solution of Problem II if either $\Gamma_{0}$ is countable or else, in the uncountable case, intersects itself only a countable number of times.

Proof. If $\Gamma_{0}=\left\{\gamma_{1}, \gamma_{2}, \cdots\right\}$, proceed inductively defining $f$ on $\gamma_{N+1}-$ $\bigcup_{n=1}^{N} \gamma_{n}$ so that $C\left(f, \gamma_{N+1}\right)=C\left(\gamma_{N+1}\right)$. This is possible because $\gamma_{N+1}$ and $\bigcup_{n=1}^{N} \gamma_{n}$ will have an empty intersection in some deleted neighborhood of the origin. The function $f$ may be defined arbitrarily off $\Gamma_{0}$.

If $\Gamma_{0}$ intersects itself countably-many times, we can write $\Gamma_{0}=\Gamma_{1} \cup \Gamma_{2}$ where $\Gamma_{1}$ is countable and $\Gamma_{2}$ is nonintersecting. Since $\Gamma_{1}$ and $\Gamma_{2}$ are solutions of Problem II and since there are no intersections between these families, $\Gamma_{0}$ is also a solution.

If a comparable family intersects itself uncountably-many times, it may or may not be a solution of Problem II.

EXAMPLE 1. $\left\{y=a x^{2}: a \in R\right\} \cup\{y=m x: m \in R\}$ is a solution.

Proof. The curve $y=x^{3 / 2}$ serves as a "dividing curve" (in the first quadrant) in the sense that all of the lines are eventually above it and all of the parabolas are eventually below it. Bettazzi's construction can now be applied.

EXAMPLE 2. $\left\{y=a x+x^{2}: a \in R\right\} \cup\left\{y=b x-x^{2}: b \in R\right\}$ is not a solution.

Proof. If the given family were a solution, there would exist a function $f$ with limiting value 0 along curves of the form $y=a x+x^{2}$ and limiting value 1 along curves of the form $y=b x-x^{2}$. Hence there would exist positive functions $r(a)$ and $s(b)$ such that $f\left(x, a x+x^{2}\right)<\frac{1}{2}$ whenever $x<r(a)$ and $f\left(x, b x-x^{2}\right)>\frac{1}{2}$ whenever $x<s(b)$. It would then follow that each of the sets $A_{n}=\{a \in R: r(a)>1 / n\}$ is nowhere dense and hence that $R$ was of the first category.

4. Unsolved problems, conjectures. By a truncation of an approach curve $\gamma$ will be meant the restriction of $\gamma$ to some deleted neighborhood 
of 0 . If every curve of a given family is truncated, the corresponding family of truncations is called a truncation of the original family. In the proofs for the above examples, we were essentially showing the existence or nonexistence of nonintersecting truncations.

Conjecture. A family $\Gamma_{0}$ is a solution of Problem II if and only if $\Gamma_{0}$ has a nonintersecting truncation.

The nonintersecting truncation problem is related to and dependent upon an even more basic problem. Consider a set $S$ of real numbers and a real-valued function $m$ defined on $S$. For $s \in S$, let $L_{s}$ denote the half-line $y=m(s) x+s, x \geqq 0$, and let $\mathscr{L}=\left\{L_{s}: s \in S\right\}$. Call $(S, m)$ acceptable if $\mathscr{L}$ has a nonintersecting truncation. "Truncation" is defined in exactly the same way as before.) Nongeometrically, this corresponds to the existence of a positive function $p$ on $S$ such that

$$
\frac{m(s)-m(t)}{t-s}<\max (p(s), p(t))
$$

whenever $s, t \in S, s \neq t$.

Conjectures. (1) $(S, m)$ is acceptable if and only if for each decomposition $(A, B)$ of $S$ there exists a positive function $p$ on $S$ such that

$$
\frac{m(a)-m(b)}{b-a}<\max (p(a), p(b))
$$

whenever $a \in A, b \in B$; (2) $(S, m)$ is acceptable for all $m$ if and only if $S$ is a set which is always of the first category [5, p. 516]; (3) $S$ is always of the first category if and only if for each decomposition $(A, B)$ of $S$ there exist sequences of sets $\left\{A_{n}\right\}$ and $\left\{B_{n}\right\}$ such that

(i) $A_{1} \subset A_{2} \subset \cdots, B_{1} \subset B_{2} \subset \cdots$,

(ii) $A=\bigcup_{n} A_{n}, B=\bigcup_{n} B_{n}$, and

(iii) $\bar{A}_{n} \cap B_{n}=\varnothing, A_{n} \cap \bar{B}_{n}=\varnothing$.

\section{REFERENCES}

1. Rodolfo Bettazzi, Sui punti di discontinuita delle funzioni di variabile reale, Rend. Palermo 6 (1892), 173-195.

2. E. F. Collingwood and A. J. Lohwater, The theory of cluster sets, Cambridge Tracts in Math. and Math. Physics, no. 56, Cambridge Univ. Press, Cambridge, 1966. MR 38 \#325.

3. O. Haupt and G. Aumann, Differential- und Integralrechnung. Vol. 2, de Gruyter, Berlin, 1938, p. 61.

4. E. W. Hobson, The theory of functions of a real variable. Vol. 1, 3rd ed., Dover, New York, 1957. 
5. K. Kuratowski, Topologie. Vol. 1, PWN, Warsaw, 1958; English transl., Academic Press, New York; PWN, Warsaw, 1966. MR 19, 873; MR 36 \#840.

6. Kiyoshi Noshiro, Cluster sets, Ergebnisse der Mathematik und ihrer Grenzgebiete, N.F., Heft 28, Springer-Verlag, Berlin, 1960. MR 24 \#A3295.

7. Arthur Rosenthal, On the continuity of functions of several variables, Math. $Z$. 63 (1955), 31-38. MR 17, 245.

Department of Mathematics, University of Nebraska, Lincoln, Nebraska 68508 